\title{
AUXILIARES E TÉCNICOS DE ENFERMAGEM NA SAÚDE DA FAMÍLIA: PERFIL SOCIODEMOGRÁFICO E NECESSIDADES DE QUALIFICAÇÃO
}

\author{
NURSING ASSISTANTS AND TECHNICIANS IN THE FAMILY HEALTH: SOCIODEMOGRAPHIC \\ PROFILE AND QUALIFICATION NEEDS
}

\author{
Francisco Rosemiro Guimarães Ximenes Neto ${ }^{1}$ \\ Márcio Clébio Fonseca Costa 2 \\ José Rocha ${ }^{3}$ \\ Isabel Cristina Kowal Olm Cunha4
}

Resumo Estudo exploratório-descritivo, realizado com 38 auxiliares e 17 técnicos de enfermagem que atuavam na Estratégia Saúde da Família (ESF), nos municípios de Acaraú, Chaval, Coreaú, Granja, Marco e Santana do Acaraú, no estado do Ceará, no período de março a abril de 2005, que objetivou traçar o perfil e as necessidades de qualificação desses profissionais. O perfil mostrou que, em sua maior parte, eram mulheres casadas, com idade entre 31 e 40 anos, ensino médio concluído, renda de um salário mínimo, atuando na área rural. Quanto às necessidades de qualificação por eles apontadas, destacam-se os temas biossegurança, internação domiciliar, métodos e técnicas de cuidados com feridas e sondas, esterilização, atividades educativas e acolhimento. A maior parte das necessidades referidas resume-se a técnicas e sugere deficiências na formação profissional. Enfatiza-se a importância de uma política de educação permanente, para melhor capacitação e melhoria na atuação deste segmento profissional na ESF.

Palavras-chave atenção primária à saúde; enfermagem; perfil; educação profissional.
Abstract An exploratory and descriptive study, conducted with 38 nursing assistants and 17 nursing technicians who worked in the Family Health Strategy (ESF), in the municipalities of Acaraú, Chaval, Coreaú, Granja, Marco and Santana do Acaraú, in the state of Ceará, from March to April of 2005. The objective was to trace the profile and qualification needs of these professionals. The profile showed that most were married women, between 31 and 40 years of age, having completed high school, receiving a minimum income, and working in the rural area. The main qualification needs they pointed out include biosafety, home care, methods and techniques of treating wounds and probes, sterilization, educational activities and receiving the patient. Most of the needs mentioned are techniques, suggesting deficiencies in professional training. The authors emphasize the importance of a permanent policy of education, to improve the qualification of this professional segment and its work in the ESF.

Keywords nursing; professional qualification; nursing assistants; public policies. 


\section{Introdução}

O processo de construção do Sistema Único de Saúde (SUS) é resultante de um conjunto de embates políticos e ideológicos, travados por diferentes atores sociais ao longo dos anos, decorrente de concepções diferenciadas, a exemplo das políticas de saúde; as formas como se organizam os serviços não são frutos apenas do momento atual, ao contrário, têm uma longa trajetória de formulações e lutas (Cunha e Cunha, 2001).

Antes do surgimento do SUS, a grande atuação do poder público nessa área se dava por meio do Instituto Nacional de Previdência Social (INPS). Este foi o resultado da fusão, em 1966, dos institutos de aposentadoria e pensões que, em 1978, por meio da Lei do Sistema Nacional de Previdência e Assistência Social (Sinpas), foi desdobrado em Instituto de Administração da Previdência Social (Iapas), INPS, e Instituto Nacional de Assistência Médica de Previdência Social (Inamps). Este último tinha a responsabilidade de prestar assistência à saúde de seus associados, o que justificava a construção de grandes unidades de atendimento ambulatorial e hospitalar, como também a contratação de serviços privados nas regiões com maior desenvolvimento econômico e nos grandes centros urbanos, onde estava a maioria dos beneficiários (Souza, 2003).

Com a crise de financiamento da Previdência Social, que começou a se manifestar em meados de 1970, o Inamps adotou várias providências para racionalizar suas despesas e começou, na década seguinte, a comprar serviços do setor público - hospitais universitários federais e redes de unidades das secretarias estaduais e municipais de saúde -, inicialmente por meio de convênios. A assistência à saúde, prestada pela rede pública, mesmo com o financiamento do Inamps, era apenas para seus beneficiários (Souza, 2003).

Esse modelo de organização da saúde era centrado no médico, num modelo curativo e hospitalar. Havia uma visão muito restrita de promoção da saúde e prevenção de doenças, e isso se dava a partir de algumas ações de saúde, como vacinação e controle de endemias.

A Constituição Federal de 1988 institucionalizou a implantação do SUS, estruturado de forma descentralizada, hierarquizada e regionalizada, de acesso universal. O parágrafo único do artigo 198 diz que o SUS será financiado com recursos próprios do orçamento da seguridade social, da União, dos estados, do Distrito Federal e dos municípios, além de outras fontes (Brasil, 1988). As ações e os serviços públicos de saúde e serviços privados contratados ou conveniados que integram o SUS são desenvolvidos de acordo com as diretrizes previstas no artigo 198, obedecendo ainda a princípios doutrinários e organizacionais que norteiam e unificam essas ações e serviços. Com a criação do SUS na Constituição Federal, a regulamentação 
por meio da lei $\mathrm{n}^{\circ} 8.080 / 1990$ e a lei $\mathrm{n}^{\circ} 8.142 / 1990$, intensifica-se o processo de descentralização do setor saúde e, conseqüentemente, a implantação e implementação de políticas, programas, serviços e ações, em âmbito estadual, e, principalmente, municipal. Com a descentralização, inicia-se a busca de mudança do modelo de atenção à saúde existente, centrado no hospital e na cura, e passam a crescer, tanto quantitativa quanto qualitativamente, as ações voltadas para a atenção primária à saúde (APS).

A organização da APS no Brasil expande-se com a criação do Programa Saúde da Família (PSF) (Brasil,1998). O PSF dissemina-se no país, deixando o formato de programa governamental e passando a política estratégica.

Em 1997, o Ministério da Saúde admite ser o PSF uma estratégia, por sua abrangência, seu impacto e resolubilidade, além de buscar a "(...) reversão do modelo assistencial vigente. Por isso, (...) sua compreensão só é possível através da mudança do objeto de atenção, forma de atuação e organização geral dos serviços, reorganizando a prática assistencial em novas bases e critérios" (Ceará, 2005), passando, então, da denominação de PSF para ESF (Estratégia Saúde da Família).

A ESF está organizada a partir de uma equipe mínima composta por enfermeiro, auxiliar de enfermagem, médico e agentes comunitários de saúde (ACS). A implantação desta estratégia em todo o país fortaleceu a interiorização de profissionais de saúde para municípios sem estrutura sanitária, onde as ações de saúde eram exercidas por sujeitos denominados de 'práticos' ou 'leigos', ou ainda por atendentes de enfermagem sem qualificação. Esta movimentação sem dúvida contribuiu para melhorias no atendimento à saúde, mas também evidenciou a necessidade constante de haver equipes preparadas com profissionais treinados e capacitados, a fim de atenderem as inúmeras demandas.

No que concerne aos profissionais de enfermagem, a ESF vem permitindo o desenvolvimento do mercado de trabalho, com a ampliação das áreas de atuação e emprego, melhoria salarial, maior empoderamento e visibilidade, e a introdução de novas práticas assistenciais e na gestão.

O auxiliar de enfermagem, profissional com nível de escolaridade de primeiro grau, encontra na ESF um novo campo de práticas, diferente daquelas exercidas nos hospitais, que ainda são predominantemente voltadas para a execução de técnicas e procedimentos, e aos poucos passa a apropriar-se de um novo território, que envolve famílias, sujeitos e comunidades. Ainda com a expansão do Projeto de Profissionalização dos Trabalhadores da Área de Enfermagem (Profae), o técnico de enfermagem, profissional em nível de segundo grau, passa a atuar aos poucos na ESF, apesar de não ser exigência legal nem normativa do Ministério da Saúde.

O preparo profissional dessas duas categorias profissionais da enfermagem para atuarem na ESF deve ser acompanhado, uma vez que ainda não 
há consenso sobre as necessidades específicas de qualificação neste cenário. Assim, a partir da evolução histórica e de implantação de políticas de saúde e de enfermagem no Brasil nos últimos 30 anos, questiona-se: Que perfis possuem os auxiliares e técnicos de enfermagem para atuar na ESF? Que necessidades de qualificação possuem estes profissionais para melhor atuar nessa estratégia? Neste contexto, o presente estudo objetiva analisar o perfil sociodemográfico e identificar as necessidades de qualificação dos auxiliares e técnicos de enfermagem da ESF.

\section{Material e método}

Trata-se de um estudo do tipo exploratório, com abordagem quantitativa, realizado nos municípios de Acaraú, Chaval, Coreaú, Granja, Marco e Santana do Acaraú, da zona norte do estado do Ceará, pertencentes ao Projeto Ações Básicas de Saúde do Ceará - Programa de Capacitação, desenvolvido pela Escola de Formação em Saúde da Família Visconde de Sabóia (EFSFVS), executora do projeto, financiado pela Secretaria de Estado da Saúde (Sesa), por meio da agência alemã Kreditanstalt für Wiederaufdau (KfW) e do Instituto Brasileiro de Qualidade Nuclear (IBQN), consultor e supervisor do projeto. O critério de escolha dos municípios para participação, ao final dos anos 1980, foi o fato de apresentarem elevada taxa de mortalidade infantil. Quanto ao município de Santana do Acaraú, a escolha deveuse ao seu pioneirismo na municipalização da saúde.

De acordo com a Secretaria de Estado da Saúde (Ceará, 2005), os municípios de Acaraú, Chaval, Coreaú, Granja, Marco e Santana do Acaraú apresentam as seguintes populações, equipes da ESF e profissionais, representados na Tabela 1.

\section{Tabela 1}

\begin{tabular}{lccc}
\hline Distribuição dos auxiliares e técnicos de enfermagem, segundo os municípios \\
\hline Municípios & $\begin{array}{c}\text { População } \\
\text { estimada }\end{array}$ & $\begin{array}{c}\text { Equipes de Saúde da } \\
\left.\text { Família ( }{ }^{\circ}\right)\end{array}$ & $\begin{array}{c}\text { Auxiliares/ técnicos de } \\
\text { enfermagem (n) }\end{array}$ \\
\hline Acaraú & 49.448 & 20 & 21 \\
Chaval & 12.340 & 10 & 3 \\
Coreaú & 20.270 & 16 & 8 \\
Granja & 49.322 & 9 & 7 \\
Marco & 20.386 & 10 & 8 \\
Santana do Acaraú & 42.588 & 15 & 8
\end{tabular}


O estudo ocorreu durante os meses de março e abril de 2005. A população, no período do estudo, era de 80 profissionais entre auxiliares e técnicos de enfermagem da ESF dos municípios de Acaraú, Chaval, Coreaú, Granja, Marco e Santana do Acaraú. A amostra compreendeu 55 profissionais de enfermagem, sendo 38 auxiliares e 17 técnicos dos referidos municípios.

Os dados foram obtidos por meio de um questionário contendo as variáveis sociodemográficas e dados de formação, bem como as necessidades de qualificação do grupo pesquisado. O questionário foi aplicado durante uma oficina, realizada nos seis municípios, onde foi discutida a importância de um diagnóstico para a construção de uma política de educação permanente para as duas categorias profissionais. Os resultados estão apresentados mediante o agrupamento em forma tabular, com a discussão dos pontos mais relevantes.

A pesquisa foi feita respeitando os aspectos éticos e legais estabelecidos pela resolução $n^{\circ}$ 196/1996, de 10 de outubro de 1996, do Conselho Nacional de Saúde (CNS), que regulamenta a prática de pesquisas envolvendo seres humanos. Os entrevistados tiveram o livre arbítrio em participar da pesquisa, fornecendo seu consentimento livre e esclarecido, onde foi certificado que não houve riscos à saúde. O sigilo e o anonimato foram garantidos. Para realização da pesquisa, obteve-se inicialmente a liberação das secretarias da saúde dos respectivos municípios.

\section{Perfil sociodemográfico}

O estudo compreendeu 55 profissionais, sendo 38 (69\%) auxiliares de enfermagem e 17 (31\%) técnicos de enfermagem. O maior número de auxiliares de enfermagem no SUS deve-se a políticas anteriores do Ministério da Saúde, que capacitou os atendentes de enfermagem transformando-os nessa categoria. Com a proibição de contratação de atendentes de enfermagem pelos serviços públicos e privados de saúde, houve um grande fluxo de profissionalização do pessoal atendente para auxiliar de enfermagem. Ainda somente a partir da metade da década de 1990, em locais prioritários, é que houve impulso para ampliar a formação de técnicos de enfermagem, para assumirem ações de maior complexidade.

Na Tabela 2 está descrito o perfil sociodemográfico dos auxiliares e técnicos de enfermagem. 
Tabela 2

Perfil sociodemográfico de auxiliares e técnicos de enfermagem da Estratégia Saúde da Família

\begin{tabular}{|c|c|c|c|c|}
\hline \multirow{2}{*}{ Variáveis } & \multicolumn{2}{|c|}{ Auxiliares } & \multicolumn{2}{|c|}{ Técnicos } \\
\hline & No & (\%) & No & (\%) \\
\hline \multicolumn{5}{|l|}{ Idade } \\
\hline 21 a 30 & 11 & 29,0 & 5 & 29,0 \\
\hline 31 a 40 & 17 & 45,0 & 9 & 53,0 \\
\hline 41 a 50 & 8 & 21,0 & 1 & 6,0 \\
\hline 50 e mais & 1 & 2,5 & - & - \\
\hline Não responderam & 1 & 2,5 & 2 & 12,0 \\
\hline Total & 38 & 100,0 & 17 & 100,0 \\
\hline \multicolumn{5}{|l|}{ Sexo } \\
\hline Feminino & 34 & 89,0 & 14 & 82,0 \\
\hline Masculino & 4 & 11,0 & 3 & 18,0 \\
\hline Total & 38 & 100,0 & 17 & 100,0 \\
\hline \multicolumn{5}{|l|}{ Estado civil } \\
\hline Casado & 16 & 42,0 & 9 & 53,0 \\
\hline Solteiro & 12 & 32,0 & 5 & 29,0 \\
\hline Com companheiro fixo & 5 & 13,0 & 3 & 18,0 \\
\hline Separado/Divorciado & 5 & 13,0 & - & - \\
\hline Total & 38 & 100,0 & 17 & 100,0 \\
\hline \multicolumn{5}{|c|}{ Renda mensal R\$ (Salário mínimo - SM*) } \\
\hline Até $1 \mathrm{SM}$ & 17 & 45,0 & 13 & 76,0 \\
\hline 2 a 3 SM & 12 & 32,0 & 2 & 12,0 \\
\hline 4 a 5 SM & 1 & 2,0 & 1 & 6,0 \\
\hline Não responderam & 8 & 21,0 & 1 & 6,0 \\
\hline Total & 38 & 100,0 & 17 & 100,0 \\
\hline \multicolumn{5}{|l|}{ Área de atuação } \\
\hline Urbana & 15 & 39,5 & 13 & 76,0 \\
\hline Rural & 18 & 47,5 & 4 & 24,0 \\
\hline Urbana e rural & 5 & 13,0 & - & - \\
\hline Total & 38 & 100,0 & 17 & 100,0 \\
\hline
\end{tabular}

* Salário mínimo em vigor no Brasil, de R\$ 300,00, no ano de 2005, equivalendo a US\$133,90, no dia 24/11/2005 (Ceará, 2005).

Quanto à idade, os sujeitos do estudo estão na faixa etária de 21 a 56 anos de idade, sendo que a maioria ( $47 \%$ ) está entre 31 e 40 anos. Esta faixa etária nos remete a uma diversidade de interpretações, voltando o olhar para a experiência de vida no que se refere à vivência social e coletiva; como também representam uma parcela significativa de trabalhadores em saúde que estão no auge de sua vida economicamente ativa.

A amostra é predominantemente feminina, tanto dos auxiliares (89\%) como dos técnicos de enfermagem $(82 \%)$. A feminilização na enfermagem não é um fenômeno novo, pois historicamente é uma profissão exercida ma- 
joritariamente por mulheres. Parece-nos que isso decorre de uma determinada concepção que entende as atividades de cuidado como uma característica supostamente inata, própria à natureza biológica das mulheres, dado que a esta são delegadas funções de cuidar desde a maternidade e a criação dos filhos, e não como uma construção social das distinções sexuais, que dão significado às relações de poder entre os homens e mulheres.

A Organização Mundial da Saúde (OMS) afirma que

“em quase todos os países as mulheres constituem a vasta maioria do pessoal de enfermagem e parteiras. Em todas as partes a enfermagem é trabalho de mulher e tem as mesmas características de outras ocupações em que predomina a mulher: baixa remuneração, condição inferior, deficientes condições de trabalho, escassas perspectivas de promoção e formação deficiente" (OMS, 1994, p. 9, tradução do autor).

Quanto a esta lógica da feminilização, própria da enfermagem, Saffiotti (1997) afirma que a sociedade delimita, com bastante precisão, os campos em que pode operar a mulher, da mesma forma como escolhe os terrenos em que pode atuar o homem.

No que concerne à renda, 87,3\% (45) possuem como única fonte seu trabalho como auxiliar ou técnico de enfermagem, sendo que $45 \%$ dos auxiliares e $76 \%$ dos técnicos recebem até um salário mínimo, daí um dos motivos da falta de estímulo para a categoria auxiliar se qualificar em técnico de enfermagem, pois, como mostra este estudo, na questão salarial, não há nenhum incentivo para a classe. Auxiliares e técnicos de enfermagem que recebam entre quatro e cinco salários mínimos são a minoria. Estes salários maiores se justificam em razão destes serem funcionários públicos federais descentralizados para os municípios, que se profissionalizaram, com o intuito de assumir novas funções no cenário municipal do SUS.

A grande maioria dos auxiliares e técnicos de enfermagem da ESF convive com uma estrutura de trabalho semelhante à dos outros profissionais deste modelo, ou seja, numa situação de precarização, não apenas quanto ao aspecto contratual - são não-concursados -, mas em condições péssimas de trabalho. Entre os múltiplos problemas, pode-se citar que o trabalho de deslocamento não apresenta segurança, há inexistência de material e de equipamentos de proteção individual, não há amparo na legislação trabalhista (sem direito a férias, $13^{\circ}$ salário, insalubridade, licença-maternidade, licença-paternidade, licença ocupacional, dentre outros). Ressaltam-se ainda os baixos salários e a inexistência de um plano de carreiras, cargos e salários.

Outro fator é o exercício de outras atividades profissionais, evidenciado em 9,1\% dos entrevistados. Mendes (2002) refere-se ao emprego 
múltiplo como um obstáculo à implantação da ESF, devido a ser esta uma política que exige dedicação total e em tempo integral. As outras atividades profissionais apontadas foram os plantões em instituições hospitalares e outras como manicure e professor.

Em relação à área de atuação, 51 \% estão distribuídos na zona urbana; $40 \%$, na zona rural; e 9\% atuam nas duas áreas. Essa realidade não necessariamente foge aos princípios da ESF, nem mesmo o da co-responsabilidade que prega o reconhecimento, pela equipe de Saúde da Família, da população adscrita, criando-se um sentimento de parceria em busca da melhoria da saúde; mesmo porque a territorialização na ESF não se baseia especificamente nas demarcações demográficas já existentes, podendo assim a área de atuação de uma equipe abranger áreas rurais ou urbanas. Quanto à condição salarial, não há diferença entre o trabalho urbano e o rural, apesar da necessidade de deslocamentos.

Com a redução dos gastos sociais, em particular na educação e na saúde, as precárias condições da rede pública de ensino e as deficiências na formação de professores, questiona-se sobre as condições objetivas para se ofertar uma educação profissional que seja capaz de responder à necessidade da prática. Isso exige investimentos em pessoal, equipamentos, estrutura, material didático e outros, não necessariamente compatíveis com as demandas mais urgentes do mercado, as quais são, em muitos casos, de caráter estritamente pragmáticos (Mendes, 2002). E esse contexto deve ser buscado na educação básica e de nível médio oferecida pelos governos nas diferentes esferas, a fim de que os cidadãos usuários desenvolvam-se tanto como profissionais quanto usuários dos serviços de atenção à saúde.

Diante do exposto, apresenta-se na Tabela 3 o perfil educacional dos trabalhadores de enfermagem investigados. Verifica-se que todos os profissionais do estudo concluíram o ensino médio, o que torna esta população, em especial, melhor preparada, uma vez que a educação, aqui retratada pelo ensino fundamental e médio, tem uma função importante na formação de cidadãos e de uma nação.

Tabela 3

Perfil educacional de auxiliares e técnicos de enfermagem da Estratégia Saúde da Família

\begin{tabular}{lcccc}
\hline \multirow{2}{*}{ Perfil educacional } & \multicolumn{2}{c}{ Auxiliares } & \multicolumn{2}{c}{ Técnicos } \\
& No & (\%) & No & (\%) \\
\hline Educação básica (ensino médio) & 28 & 74,0 & 16 & 94,0 \\
Ensino superior incompleto (Pedagogia, Letras, & 8 & 21,0 & - & - \\
Química, Biologia) & & & 1 & 6,0 \\
Ensino superior completo (Pedagogia) & 2 & 5,0 & 17 & 100,0 \\
Total & 38 & 100,0 & &
\end{tabular}

Fonte: Os autores. 
Antes disso, porém, trata-se de concebê-la como direito subjetivo de todos e o espaço social de organização, produção e apropriação dos conhecimentos mais avançados produzidos pela humanidade. Mas não é essa a ênfase dada à educação básica há alguns anos. Em face das desigualdades de capital humano, houve ênfase nas concepções da educação básica ligadas à noções de empregabilidade marcada pelo viés economicista, fragmentário e tecnicista. E é nesse embate de concepções de sociedade e trabalho que se insere a disputa pela educação como uma prática social mediadora do processo político, ideológico e cultural (Frigotto, Ciavatta e Ramos, 2005).

É nesse horizonte de ensino politécnico ou tecnológico (Ferretti, 1999) que estão inseridos e são formados os auxiliares e técnicos de enfermagem, o que exige mudanças, para que esse ensino passe a articular os conceitos de cidadania com os da formação específica. Não se trata aí de uma questão linear com o mercado de trabalho, mas mediada, sem o que não se cumprem os dois imperativos: o de justiça social e acompanhamento das transformações técnico-científicas do mundo do trabalho (Frigotto, Ciavatta e Ramos, 2005).

O aperfeiçoamento educacional dos níveis básico e superior se dá na perspectiva do ingresso no mercado de trabalho. Quanto ao nível de escolaridade dos entrevistados, $100 \%$ concluíram o nível médio e $11 \%$ estão concluindo, ou concluíram, o ensino superior. Pode-se afirmar que há um paradoxo nesse contexto, pois, dessa amostra, $5 \%$ (dois) dos auxiliares e $6 \%$ (um) dos técnicos possuem nível superior e nem por isso atuam na sua área de graduação, podendo-se inferir que, se o buscaram, parece ter sido mais para fins de qualificação pessoal do que para uma busca de ascensão profissional. Todavia, há que se destacar que, como a maior parte desta formação foi voltada à área de pedagogia, fica então essa massa de trabalhadores qualificada à disposição para exercerem suas funções na ESF, bastante relacionadas com educação e saúde.

Quanto à qualificação profissional, Takahashi e Oguisso afirmam que é

“entendida como uma relação social construída pela interação dos agentes sociais em torno da propriedade, significado e uso dos conhecimentos, saberes, competências, habilidades e aptidões profissionais construídos ao longo da vida, necessários ao exercício de uma profissão, ocupação ou atividade de trabalho" (Takahashi e Oguisso, 2005, p. 39).

As necessidades de qualificação profissional dos auxiliares e técnicos de enfermagem estão descritas na Tabela 4. 
Necessidades de qualificação de auxiliares e técnicos de enfermagem da Estratégia Saúde da Família

\begin{tabular}{|c|c|c|c|c|}
\hline \multirow{2}{*}{ Necessidades de qualificação } & \multicolumn{2}{|c|}{ Auxiliares } & \multicolumn{2}{|c|}{ Técnicos } \\
\hline & $\mathrm{N}^{\circ}$ & (\%) & No & $(\%)$ \\
\hline \multicolumn{5}{|l|}{ Procedimentos } \\
\hline Métodos e técnicas de sondas & 4 & 11,0 & 7 & 41,0 \\
\hline Técnica de verificação de sinais vitais & 6 & 16,0 & 4 & 24,0 \\
\hline Métodos e técnicas de cuidado com feridas & 5 & 13,0 & 3 & 18,0 \\
\hline Métodos e técnicas de esterilização & 1 & 2,5 & 7 & 41,0 \\
\hline Métodos e técnicas de administração de medicamentos & 2 & 5,0 & 4 & 24,0 \\
\hline Técnica de verificação de medidas antropométricas & 3 & 8,0 & 1 & 6,0 \\
\hline \multicolumn{5}{|l|}{ Não responderam } \\
\hline \multicolumn{5}{|l|}{ Qualificaçōes específicas } \\
\hline Internamento domiciliar & 16 & 42,0 & 5 & 29,0 \\
\hline Biossegurança & 7 & 18,0 & 12 & 71,0 \\
\hline Acolhimento & 9 & 24,0 & 4 & 24,0 \\
\hline Métodos e técnicas de vacinação & 8 & 21,0 & 5 & 29,0 \\
\hline Visitas domiciliares & 8 & 21,0 & 3 & 18,0 \\
\hline Primeiros socorros na comunidade & 5 & 13,0 & 4 & 24,0 \\
\hline Cuidados com o recém-nascido & 3 & 8,0 & 4 & 24,0 \\
\hline Atividades educativas & 2 & 5,0 & 6 & 35,0 \\
\hline Rotinas de serviço & 2 & 5,0 & 1 & 6,0 \\
\hline $\begin{array}{l}\text { Atenção aos portadores hipertensão arterial e } \\
\text { diabetes mellitus }\end{array}$ & 1 & 2,5 & 1 & 6,0 \\
\hline Atenção à saúde da mulher & 1 & 2,5 & - & - \\
\hline Cuidados com a gestante & 1 & 2,5 & - & - \\
\hline
\end{tabular}

Fonte: Os autores.

O Ministério da Saúde está propondo a educação permanente como política de transformação das práticas de formação, de atenção, de gestão, de formulação de políticas, de participação popular e de controle social no setor da saúde. Essa educação se baseia na aprendizagem significativa, que pode ser entendida como aprendizagem-trabalho, ou seja, ela acontece no cotidiano das pessoas e das organizações (Brasil, 2005).

Essa aprendizagem acontece a todo o momento em que nos questionamos sobre algum problema atual e procuramos a resposta, a partir de uma lógica de necessidade de aprendizagem. O primeiro passo para organizar essa questão de educação permanente é procurar perceber quais as necessidades de aperfeiçoamento relativo ao nosso cotidiano, dentro da realidade do nível de atenção à saúde na qual estamos inseridos.

A partir da pesquisa pode-se compreender o universo das ações de que os profissionais da ESF sentem necessidade de aperfeiçoamento. Foram citadas, por esses profissionais, 19 ações de enfermagem que não se sentem seguros ao realizar. Se, por um lado, parece ser preocupante tal índice de 
insegurança, por outro, há que se destacar que este grupo percebe suas deficiências. Freire (2002) já mencionava que a reflexão crítica sobre a prática é fundamental, pois pensando criticamente a prática de hoje, ou de ontem, é que se pode melhorar a próxima prática.

Das 19 necessidades de educação permanente, mais da metade é ação específica da ideologia de promoção à saúde. Vale salientar que a citada com maior freqüência foi o internamento domiciliar. Isso parece refletir o interesse desses profissionais em prestar o atendimento em domicílio e também a preocupação de receberem bem a comunidade na Unidade Básica de Saúde (UBS). A necessidade de capacitação de biossegurança também retrata a insistente preocupação desses trabalhadores com eles mesmos. Nasce aí o princípio do cuidar do cuidador.

Deve ser dado destaque que, no tocante à necessidade de capacitação de procedimentos técnicos de enfermagem, houve uma porcentagem bem maior entre os técnicos do que entre os auxiliares de enfermagem. É possível inferir-se que o nível técnico tem maior conhecimento e escolaridade e estaria mais preparado para perceber suas deficiências e necessidades de melhoria. Além disso, em sua formação, o técnico amplia o limite de ação para além da abordagem mais tecnicista, incluindo princípios relacionados à administração da unidade onde se dão as ações de saúde.

Não podemos esquecer que a proposta de educação permanente é uma política pública do SUS. Para que se consiga um novo modo de organização da Atenção Básica, precisa-se de um novo perfil de trabalho e de trabalhadores. Para que essas necessidades sejam supridas, o Ministério da Saúde (Brasil, 2005) criou os pólos de educação permanente, que têm o objetivo de: formular e integrar ações de educação em serviço ou formação para usuários, dirigentes de ações ou unidades, gestores públicos, professores, estudantes e trabalhadores da área da saúde, agentes sociais e parceiros intersetoriais; provocar processos de transformação das práticas de saúde e educação na saúde; formular políticas de formação e desenvolvimento em bases locorregionais.

A Política Nacional de Atenção Básica (Brasil, 2006, p. 42) apresenta como atribuições do auxiliar e do técnico de enfermagem o seguinte: participar das atividades de assistência básica realizando procedimentos regulamentados no exercício de sua profissão na USF e, quando indicado ou necessário, no domicílio e/ou nos demais espaços comunitários (escolas, associações etc.); realizar ações de educação em saúde a grupos específicos e a famílias em situação de risco, conforme planejamento da equipe; e participar do gerenciamento dos insumos necessários para o adequado funcionamento da USF.

A referida política estabelece as atribuições do auxiliar e do técnico de enfermagem basicamente em três categorias: a técnica, centrada em 
procedimentos; a relacionada à educação em saúde; e a referente ao controle de insumos. Isto posto, pode-se inferir que o desenvolvimento de ações voltadas à promoção da saúde não foi especificamente contemplado neste contexto.

\section{Considerações finais}

O perfil dos auxiliares e técnicos de enfermagem da Estratégia Saúde da Família pesquisada mostrou que, em sua maior parte, é de mulheres casadas, com idades entre 31 e 40 anos, com ensino médio concluído, renda de um salário mínimo, atuando na área rural. Quanto às necessidades de qualificação por eles apontadas, destacam-se biossegurança, internação domiciliar, métodos e técnicas de cuidado com feridas e sondas, esterilização, atividades educativas e acolhimento.

O estudo delineou carências significativas no grupo de auxiliares e técnicos de enfermagem, em relação a atualizações e treinamentos. Isto se deve certamente a lacunas na formação, formação tardia e não atualização após a entrada no mercado de trabalho, fato possível de reversão se houver treinamentos adequados. As instituições empregadoras devem, assim, disponibilizar oportunidades para que estes profissionais possam ter acesso à informação e consigam acompanhar toda a evolução técnico-científica que se vivencia hoje na área da saúde. Outra lacuna percebida foi, especialmente, em relação aos conteúdos específicos da Estratégia Saúde da Família e aos princípios do SUS. Assim, deve ser tratada como estratégia de qualificação de pessoas a política de educação permanente hoje em vigor, a fim de que por meio de estímulos por parte dos gestores públicos estes profissionais sejam capazes de desempenhar suas funções na atenção básica de forma competente e resolutiva, com ações de promoção, prevenção, recuperação e reabilitação em saúde e que possam participar ativamente da construção do SUS.

Ainda é algo relevante a não diferenciação das atividades exercidas entre os auxiliares e técnicos de enfermagem dentro da Estratégia Saúde da Família, pois não se evidenciou uma distinção entre estas, apesar de todas estarem de acordo com o que é preconizado pelo Ministério da Saúde. Vale ressaltar que a diferenciação entre o técnico e o auxiliar é basicamente a escolaridade e o nível de complexidade das ações, em que o primeiro desenvolve tanto ações pertinentes ao auxiliar de enfermagem quanto aquelas que necessitam de uma maior qualificação. Todavia, atualmente há um movimento do conselho profissional no sentido de qualificar todos os auxiliares, transformando-os em técnicos de enfermagem, pois se acredita que 
isso repercutiria em melhoria da qualidade da assistência de enfermagem prestada à clientela.

Quer o olhar pouse sobre técnicos ou sobre os auxiliares de enfermagem, é constatado que estas categorias profissionais necessitam de organização como classe trabalhadora, para lutar por melhores condições salariais e vínculos empregatícios justos e que atendam à lei. Sem a formação de entidades de classe e informação no que se refere a direitos e deveres, acredita-se que não haverá alteração nas situações de precarização do trabalho e ao acesso à garantia dos direitos como cidadãos trabalhadores.

O desafio para esta categoria está, pois, visível e deve ser discutido. Os gestores da área da saúde necessitam compartilhar experiências com resultados positivos para a inclusão destas duas categorias.

\section{Notas}

1 Professor auxiliar da Universidade Estadual do Vale do Acaraú (Uva), Sobral, Ceará, Brasil. Mestre em Saúde Pública pela Universidade Estadual do Ceará (Uece). $<$ rosemironeto@gmail.com>

Correspondência: Rua Sebastião Miranda, s/n, Centro, Cariré, Ceará, Brasil, CEP 62184-000.

2 Enfermeiro da Prefeitura Municipal de Acaraú, Ceará, Brasil. Graduado em Enfermagem pela Universidade Estadual Vale do Acaraú (Uva). < marcioclebio@gmail.com>

3 Enfermeiro da Secretaria Municipal da Saúde de São Paulo, Brasil. Mestre em Estudos Avançados da Educação pela Universidade de Guarulhos (UnG). <prof.jose.rocha@hotmail.com>

4 Professora adjunta da Universidade Federal de São Paulo (Unifesp), São Paulo, Brasil. Doutora em Saúde Pública pela Universidade de São Paulo (USP). <icris@denf.epm.br>

\section{Referências}

BRASIL. Lei $n^{\circ}$ 7.498/1986. Lei do Exercício Profissional. Dispõe sobre o exercício profissional da enfermagem. Diário Oficial da República Federativa do Brasil, Poder Executivo, Brasília, DF, 26 jun. 1986. Disponível em: <http://corensp.org.br/072005/> Acesso em: 12 mar. 2008.
BRASIL. Constituição da República Federativa do Brasil. Rio de Janeiro: Rio Condor, 1988.

BRASIL. Ministério da Saúde. Secretaria de Assistência à Saúde. Coordenação de Saúde da Comunidade. Saúde da Família: uma 
estratégia para reorientação do modelo assistencial. 2. ed. Brasília: MS, 1998.

BRASIL. Ministério da Saúde. A educação permanente entra na roda: pólos de educação permanente em saúde - conceitos e caminhos a percorrer. Brasília: MS, 2005. 36p. (Série C. Projetos, Programas e Relatórios) (Educação na Saúde).

BRASIL. Ministério da Saúde. Secretaria de Atenção à Saúde. Departamento de Atenção Básica. Política nacional de atenção básica. Brasília: MS, 2006. 60 p. (Série A. Normas e Manuais Técnicos) (Série Pactos pela Saúde 2006 , v. 4).

CEARÁ. Secretaria da Saúde do Estado. Dados e estatísticas. Disponível em: $<$ www.saude.ce.gov.br $>$. Acesso em: 20 jul. 2005.

CUNHA, João Paulo; CUNHA, Rosani Evangelista. Sistema Único de Saúde: princípios. In: FERRETTI, Celso João. Formação profissional e reforma do ensino técnico no Brasil: anos 90. Educação e Sociedade, a. XVIII, n. 59, ago. 1999.

FREIRE, Paulo. Pedagogia da autonomia: saberes necessários à prática educativa. São Paulo: Paz e Terra, 2002.
FRIGOTTO, Gaudêncio; CIAVATTA, Maria; RAMOS, Marise. Ensino médio integrado: concepção e contradições. São Paulo: Cortez, 2005. 175p.

MENDES, Eugênio Vilaça. A atenção primária à saúde no SUS. Fortaleza: Escola de Saúde Pública do Ceará, 2002. 92 p.

ORGANIZACIÓN MUNDIAL DE LA SALUD. La Enfermería más allá del año 2000: Informe de un grupo de estudio de la OMS. OMS: Ginebra, 1994. (OMS, Serie de informes técnicos; 842).

SAFFIOTI, Heleieth I. Bongiovani. A mulher na sociedade de classes: mito e realidade. Petrópolis: Vozes, 1997.

SOUZA, Heloiza Machado de. Análise das práticas assistenciais na área de Saúde da Família: um estudo de caso em estado selecionado. In: PIERANTONI, Celia Regina; VIANA, Cid Manso M. (Org.). Gestão de Sistemas de Saúde. 1. ed. Rio de Janeiro: Segrecar, 2003, v. 1, 389 p.

TAKAHASHI, Regina Tashie; OGUISSO, Taka. Reflexões sobre a certificação profissional de Enfermagem no Brasil. Revista Paulista de Enfermagem, v. 24, n. 4, out.dez. 2005. p. 37-42.

Recebido em 04/10/2007

Aprovado em 03/04/2008 